\title{
ADIABATIC PHASES FROM AN EFFECTIVE ACTION
}

\author{
Dieter W. DÜSEDAU \\ Randall Laboratory of Physics, University of Michigan, Ann Arbor, MI 48109-1120, USA \\ Received 4 February 1988
}

Berry's phase is calculated as a term in the derivative expansion of the effective action of a spin- $\frac{1}{2}$ system in an external field.

A quantum mechanical system depending on an external parameter, taken slowly around a closed loop in parameter space exhibits in addition to the familiar dynamical phase variation a geometric phase factor [1]. In this short note we show that this phase factor occurs naturally in the derivative expansion of the effective action [2]: Berry's phase can be calculated by straightforward and direct evaluation of a functional determinant.

We shall concentrate on the ground state of a spin- $-\frac{1}{2}$ system in an external magnetic field $\boldsymbol{B}$ with lagrangian $[2,3]$

$L=\frac{1}{2} \mathrm{i}\left(\xi^{n} \dot{\xi}^{n}+\epsilon_{m n j} \xi^{m} \xi^{n} B^{j}\right)$,

where the $\xi$ 's are real anticommuting variables. To obtain the effective action $\Gamma(\boldsymbol{B})$ we have to evaluate the path integral

$\exp [\mathrm{i} \Gamma(B)]=\int\left[\mathrm{D} \xi^{n}\right] \exp \left(\mathrm{i} \int \mathrm{d} t\left[\frac{1}{2} \mathrm{i}\left(\xi^{n} \dot{\xi}^{n}+\epsilon_{m n j} \xi^{m} \xi^{n} B^{j}\right)\right]\right)$,

which is easily done, since $L$ is only quadratic in the $\xi$ 's. We learn that

$\exp [\mathrm{i} \Gamma(B)]=\operatorname{det}^{1 / 2}\left(\omega \delta_{m n}+\mathrm{i} \epsilon_{m n j} B^{j}\right)$

or

$\Gamma(\boldsymbol{B})=-\frac{1}{2} \mathrm{i} \operatorname{Tr} \ln \left(\omega \delta_{m n}+\mathrm{i} \epsilon_{m n j} B^{j}\right)$,

where $\omega$ is the energy operator and the trace Tr is understood in a functional sense. Gerbert [3] arrives at the same expression for $\Gamma(B)$, (3), only he goes on to evaluate $\Gamma$ making strong use of symmetry principles. We shall instead evaluate (4) in a straightforward way. Since $\boldsymbol{B}(t)$ is time dependent, the operator $\left(\omega \delta_{m n}+\mathrm{i} \epsilon_{m n} B^{j}\right)$ is not diagonal in the energy representation, and (4) cannot be evaluated exactly. Instead we may expand the density of $\Gamma(\boldsymbol{B})$ in powers of $\dot{\boldsymbol{B}}[4,5]$. Schematically, we may write

$\Gamma(\boldsymbol{B})=\int \mathrm{d} t\left[-V_{\text {eff }}(\boldsymbol{B})+\boldsymbol{A}(\boldsymbol{B}) \dot{B}+\frac{1}{2} \mathrm{i} \dot{B}^{i} M_{i j} \dot{B}^{j}+\ldots\right]$.

Clearly $V_{\text {eff }}(B)$ is just the effective potential and $A(B)$ Berry's connection. $V_{\text {eff }}(B)$ can be evaluated by taking $\boldsymbol{B}(t)=\boldsymbol{B}_{0}$ to be a constant $[5,6]$. Then (4) reduces to an integral in momentum space: 


$$
\begin{aligned}
& \Gamma\left(B_{0}\right)=-\frac{1}{2} \mathrm{i} \operatorname{Tr} \ln \left(\omega \delta_{m n}+\mathrm{i} \epsilon_{m n j} B_{0}^{j}\right)=-\frac{1}{2} \mathrm{i} \int \mathrm{d} t \int \frac{\mathrm{d} \omega}{2 \pi} \ln \left[\omega^{3}\left(1-B_{0}^{2} / \omega^{2}\right)\right] \\
& \quad=\frac{1}{2} \int \mathrm{d} t \int \frac{\mathrm{d} \omega_{\mathrm{E}}}{2 \pi} \ln \left(1+B_{0}^{2} / \omega_{\mathrm{E}}^{2}\right)=\int \mathrm{d} t \frac{B_{0}}{2},
\end{aligned}
$$

where we subtracted an infinite constant and made the substitution $\omega=\mathrm{i} \omega_{\mathrm{E}}$. Comparing (5) and (6) we learn that $V_{\text {eff }}(B)=-\frac{1}{2} B=-\frac{1}{2} \sqrt{\boldsymbol{B}^{2}}$, which is the standard ground state energy $\# 1$.

To evaluate the higher order terms in (5) we employ a technique due to Fraser [5]; set $B^{i}(t)=B_{0}^{i}+\tilde{B}^{i}(t)$, where $\boldsymbol{B}_{0}$ is again a constant background, and expand (4) in powers of $\widetilde{B}^{i}$ and $\widetilde{B}^{i}$. This expansion around $\boldsymbol{B}_{0}$ is then compared with the corresponding expansion of (5), and the coefficients $A$ and $M_{i j}$ are identified. Let us first expand (5) to second order in $\widetilde{B}^{i}$ :

$$
\begin{aligned}
\Delta & =\Gamma\left(B_{0}^{i}+\tilde{B}^{i}\right)-\Gamma\left(B_{0}^{i}\right) \\
& =\int \mathrm{d} t\left(-\tilde{B}^{i} \frac{\partial V}{\partial B_{0}^{i}}-\frac{1}{2} \widetilde{B}^{i} \tilde{B}^{j} \frac{\partial^{2} V}{\partial B_{0}^{i} \partial B_{0}^{j}}+\tilde{B}^{i} A_{i}\left(B_{0}\right)+\tilde{B}^{i} \frac{\partial A_{i}}{\partial B_{0}^{j}} \tilde{B}^{j}+\frac{1}{2} \tilde{B}^{i} \tilde{B}^{j} M_{i j}\left(B_{0}\right)+\ldots\right) .
\end{aligned}
$$

From (4) we also know that

$$
\begin{aligned}
\Delta & =-\frac{1}{2} \mathrm{i} \operatorname{Tr}\left\{\ln \left[\omega \delta_{m n}+\mathrm{i} \epsilon_{m n j}\left(B_{0}^{j}+\tilde{B}_{0}^{j}\right)\right]-\ln \left(\omega \delta_{m n}+\mathrm{i} \epsilon_{m n j} B_{0}^{j}\right)\right\} \\
& =-\frac{1}{2} \mathrm{i} \operatorname{Tr} \ln \left[\delta_{m l}+\mathrm{i} \epsilon_{m n j} \widetilde{B}^{j}\left(\omega \delta^{n l}-B_{0}^{n} B_{0}^{l} / \omega-\mathrm{i} \epsilon^{n l k} B_{0}^{k}\right) /\left(\omega^{2}-B_{0}^{2}\right)\right] .
\end{aligned}
$$

Expanding the logarithm in (8) generates a power series in $\widetilde{B}^{i}$, which we later may compare with (7)

$\Delta=-\frac{1}{2} \mathrm{i} \operatorname{Tr}\left(\frac{\widetilde{B}^{j} \epsilon_{m n j} \epsilon^{n m k} B_{0}^{k}}{\omega^{2}-B_{0}^{2}}+\frac{1}{2} \epsilon_{m n j} \widetilde{B}^{j} \frac{\omega \delta^{n l}-B_{0}^{n} B_{0}^{l} / \omega-\mathrm{i} \epsilon^{n l k} B_{0}^{k}}{\omega^{2}-B_{0}^{2}} \epsilon_{l p q} \widetilde{B}^{q} \frac{\omega \delta^{p m}-B_{0}^{p} B_{0}^{m} / \omega-\mathrm{i} \epsilon^{p m r} B_{0}^{r}}{\omega^{2}-B_{0}^{2}}\right)$.

Here, the term linear in $\widetilde{B}^{i}$ is just the first derivative of the effective potential, and for the calculation of $A$ irrelevant. It comes as no surprise to us that formula (9) misses the term $\widetilde{B}^{i} A_{i}\left(B_{0}\right)$, which is present in (7), since it is a total derivative. After some algebra and using the cyclicity of the trace the term quadratic in $\widetilde{B}^{i}$ may be rewritten as

$$
\begin{gathered}
\Delta^{(2)}=-\frac{1}{2} \mathrm{i} \operatorname{Tr}\left(-\tilde{B}^{j} \frac{\omega}{\omega^{2}-B_{0}^{2}} \tilde{B}^{j} \frac{\omega}{\omega^{2}-B_{0}^{2}}-\tilde{B} \cdot B_{0} \frac{\omega}{\omega^{2}-B_{0}^{2}} \tilde{B} \cdot B_{0} \frac{1 / \omega}{\omega^{2}-B_{0}^{2}}+B_{0}^{2} \tilde{B}^{j} \frac{\omega}{\omega^{2}-B_{0}^{2}} \tilde{B^{j}} \frac{1 / \omega}{\omega^{2}-B_{0}^{2}}\right. \\
\left.-\mathrm{i} \epsilon_{j l k} B_{0}^{k} \tilde{B}^{j} \frac{\omega}{\omega^{2}-B_{0}^{2}} \tilde{B}^{\prime} \frac{1}{\omega^{2}-B_{0}^{2}}+\mathrm{i} \epsilon_{l k j} B_{0}^{2} B_{0}^{k} \tilde{B}^{j} \frac{1 / \omega}{\omega^{2}-B_{0}^{2}} \tilde{B}^{\prime} \frac{1}{\omega^{2}-B_{0}^{2}}-\tilde{B} \cdot B_{0} \frac{1}{\omega^{2}-B_{0}^{2}} \tilde{B} \cdot B_{0} \frac{1}{\omega^{2}-B_{0}^{2}}\right) .
\end{gathered}
$$

Next, we will have to evaluate the trace. We can use the identity [5]

$$
\left[\tilde{B}^{i}, \frac{1}{\omega^{2}-m^{2}}\right]=\frac{1}{\left(\omega^{2}-m^{2}\right)^{2}}\left[\omega^{2}, \tilde{B}^{i}\right]+\frac{1}{\left(\omega^{2}-m^{2}\right)^{3}}\left[\omega^{2},\left[\omega^{2}, \tilde{B}^{i}\right]\right]+\ldots
$$

to move all the operators $\omega$ to the right and all the $\widetilde{B}^{i}$ to the left. Then the traces can be performed independently. The commutators needed can be evaluated to be

$$
\left[\omega, \widetilde{B}^{i}\right]=\mathrm{i} \tilde{B}^{i}, \quad\left[\omega^{2}, \widetilde{B}^{i}\right]=\widetilde{B}^{i}+2 \mathrm{i} \omega \tilde{B}^{i},\left[\omega^{2},\left[\omega, \widetilde{B}^{i}\right]\right]=\mathrm{i} \ddot{B}^{i}-2 \omega \tilde{B}^{i}, \quad\left[\omega^{2},\left[\omega^{2}, \widetilde{B}^{i}\right]\right]=\widetilde{B}^{i}+4 \mathrm{i} \omega \widetilde{B}^{i}-4 \omega^{2} \widetilde{B}^{i},
$$

To find Berry's connextion $\boldsymbol{A}(\boldsymbol{B})$ we need only collect terms with one time derivative. Commuting all $\omega$ 's to the right and collecting only the terms containing one time derivative we obtain

\footnotetext{
\#1 Depending on the sign of $i \epsilon$ in the propagator $1 /\left(\omega^{2} \pm i \epsilon\right)$ we calculate either the ground state energy or the energy of the excited state.
} 


$$
\begin{aligned}
& \Delta_{i}^{(2)}=-\frac{1}{2} \mathrm{i} \operatorname{Tr}\left(-\frac{2 \mathrm{i} \omega}{\left(\omega^{2}-B_{0}^{2}\right)^{2}}(\tilde{B} \tilde{B})+\frac{\mathrm{i} / \omega}{\omega^{2}-B_{0}^{2}}(\tilde{B} \tilde{B})-\frac{4 \mathrm{i} \omega}{\left(\omega^{2}-B_{0}^{2}\right)^{3}}\left(\tilde{B} B_{0}\right)\left(\tilde{B} B_{0}\right)\right. \\
& \left.+\frac{1 / \omega}{\omega^{2}-B_{0}^{2}}\left(\tilde{B} B_{0}\right)\left(\tilde{B} B_{0}\right)+\frac{2}{\left(\omega^{2}-B_{0}^{2}\right)^{2}} \epsilon_{j l k} \tilde{B}^{j} \hat{B}^{\prime} B_{0}^{k}\right) .
\end{aligned}
$$

Note that the first four terms in (13) can be rewritten as a time derivative. Since the trace involves an integral over time we discard them. Then

$\Delta_{1}^{(2)}=-\frac{1}{2} \mathrm{i} \operatorname{Tr}\left(\frac{2}{\left(\omega^{2}-B_{0}^{2}\right)^{2}} \epsilon_{i j k} \tilde{B}^{i} \tilde{B}^{j} B_{0}^{k}\right)=-\frac{1}{2} \mathrm{i} \int \frac{\mathrm{d} \omega}{2 \pi} \frac{2}{\left(\omega^{2}-B_{0}^{2}\right)^{2}} \int \mathrm{d} t \epsilon_{i j k} \tilde{B}^{i} \tilde{B}^{j} B_{0}^{k}=\int \mathrm{d} t \frac{1}{4} \epsilon_{i j k} \frac{B_{0}^{k}}{B_{0}^{3}} \tilde{B}^{j} \tilde{B}^{i}$.

Our final expression (14) must now be compared with the corresponding term in (7). From there we obtain

$$
\Delta_{\mathrm{I}}^{(2)}=\int \mathrm{d} t \tilde{B}^{i} \frac{\partial A_{i}}{\partial B^{j}} \widetilde{B}^{j}=\frac{1}{2} \int \mathrm{d} t\left(\frac{\partial A_{i}}{\partial B_{j}}-\frac{\partial A_{j}}{\partial B_{i}}\right) \tilde{B}^{i} \tilde{B}^{j},
$$

where the second line follows by partial integration.

Equating (14) and (15) we finally learn that

$$
\frac{1}{2} \int \mathrm{d} t\left(\frac{\partial A_{j}}{\partial B_{i}}-\frac{\partial A_{i}}{\partial B_{j}}\right) \tilde{B}^{j} \tilde{B}^{i}=\int \mathrm{d} t \frac{1}{4} \epsilon_{i j k} \frac{B_{0}^{k}}{B_{0}^{3}} \tilde{B}^{j} \tilde{B}^{i}
$$

or

$\left(\frac{\partial A_{j}}{\partial B_{i}}-\frac{\partial A_{i}}{\partial B_{j}}\right)=\frac{1}{2} \epsilon_{i j k} \frac{B_{0}^{k}}{B_{0}^{3}}$

From (17) we see immediately that $\nabla_{B} \times A=\frac{1}{2} B / B^{3}$, where $A$ is the potential of a magnetic monopole with strength $\frac{1}{2}$. The curvature $\nabla_{B} \times A$ is just the field of a magnetic monopole, as Berry's analysis [1] has shown. Hence, this method can indeed be used to evaluate the adiabatic phase directly.

The higher order terms in (5) can be obtained by the same methods, one only has to collect terms with more time derivatives. Since there are no matters of principle involved and they are calculated elsewhere [3], we omit the calculation here.

I would like to thank O. Cheyette, R. Jackiw and J. Lott for discussions.

\section{References}

[1] M.V. Berry, Proc. R. Soc. London A 392 (1984) 45.

[2] R. Jackiw, private communication; MIT preprint CTP\#1529 (October 1987).

[3] Ph. Gerbert, MIT preprint CTP\#1537 (October 1987).

[4] R. Akhoury and Y.-P. Yao, Phys. Rev. D 25 (1982) 3361.

[5] C.M. Fraser, Z. Phys. C 28 (1985) 101.

[6] R. Jackiw, Phys. Rev. D 9 (1974) 1686. 